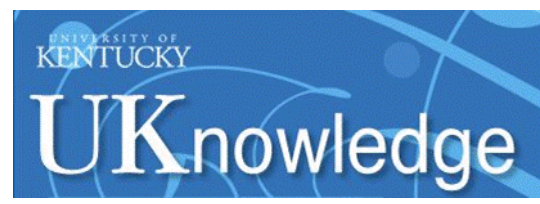

University of Kentucky

UKnowledge

$4-18-2013$

\title{
Strongly Localized Magnetization Modes in Permalloy Antidot Lattices
}

\author{
J. Sklenar \\ Northwestern University \\ V. S. Bhat \\ University of Kentucky \\ Lance E. De Long \\ University of Kentucky, delong@pa.uky.edu \\ O. Heinonen \\ Northwestern University \\ J. B. Ketterson \\ Northwestern University
}

Follow this and additional works at: https://uknowledge.uky.edu/physastron_facpub

Part of the Astrophysics and Astronomy Commons, and the Physics Commons

Right click to open a feedback form in a new tab to let us know how this document benefits you.

\section{Repository Citation}

Sklenar, J.; Bhat, V. S.; De Long, Lance E.; Heinonen, O.; and Ketterson, J. B., "Strongly Localized Magnetization Modes in Permalloy Antidot Lattices" (2013). Physics and Astronomy Faculty Publications. 238.

https://uknowledge.uky.edu/physastron_facpub/238

This Article is brought to you for free and open access by the Physics and Astronomy at UKnowledge. It has been accepted for inclusion in Physics and Astronomy Faculty Publications by an authorized administrator of UKnowledge. For more information, please contact UKnowledge@lsv.uky.edu. 


\section{Strongly Localized Magnetization Modes in Permalloy Antidot Lattices}

\section{Digital Object Identifier (DOI)}

http://dx.doi.org/10.1063/1.4802278

\section{Notes/Citation Information}

Published in Applied Physics Letters, v. 102, article 152412, p. 1-4.

Copyright 2013 American Institute of Physics. This article may be downloaded for personal use only. Any other use requires prior permission of the author and the American Institute of Physics.

The following article appeared in Applied Physics Letters, v. 102, article 152412, p. 1-4 and may be found at http://dx.doi.org/10.1063/1.4802278. 


\title{
Strongly localized magnetization modes in permalloy antidot lattices
}

\author{
J. Sklenar, ${ }^{1}$ V. S. Bhat, ${ }^{2}$ L. E. DeLong, ${ }^{2}$ O. Heinonen, ${ }^{1,3}$ and J. B. Ketterson ${ }^{1,4}$ \\ ${ }^{1}$ Department of Physics and Astronomy, Northwestern University, Evanston, Illinois 60208, USA \\ ${ }^{2}$ Department of Physics, University of Kentucky, Lexington, Kentucky 40508, USA \\ ${ }^{3}$ Materials Science Division, Argonne National Laboratory, Argonne, Illinois 60515, USA \\ ${ }^{4}$ Department of Electrical and Computer Engineering, Northwestern University, Evanston, Illinois 60515, USA
}

(Received 28 August 2012; accepted 5 April 2013; published online 18 April 2013)

\begin{abstract}
Antidot lattices (ADLs) patterned into soft magnetic thin films exhibit rich ferromagnetic resonance (FMR) spectra corresponding to many different magnetization modes. One of the predicted modes is highly localized at the edges of the antidots; this mode is difficult to detect experimentally. Here we present FMR data for a permalloy thin film patterned into a square array of square antidots. Comparison of these data with micromagnetic simulations permits identification of several edge modes. Our simulations also reveal the effect of the antidot shape on the mode dispersion. @ 2013 AIP Publishing LLC [http://dx.doi.org/10.1063/1.4802278]
\end{abstract}

The emerging field of magnonics is concerned with the generation and manipulation of spin waves. Magnonic crystals (MCs) are magnetic structures with periodic perturbations or variations in a magnetic property of the system. MC can serve as functional media for exciting and processing spin waves, ${ }^{1-3}$ and one- and two-dimensional MC have been studied previously. ${ }^{4,5}$ One MC prototype is the two-dimensional antidot lattice (ADL) which is a periodic array of antidots (holes) in a FM thin film. ADL display a rich variety of magnetic excitations ${ }^{1,6}$ including extended modes that reside between rows of antidots (ADs), as well as localized modes, all exhibiting the Bloch translational symmetry of the ADL.

Initial experimental work that hinted at the existence of localized modes utilized ferromagnetic resonance (FMR) techniques and time resolved Kerr microscopy (TRKM).,8 Simulations performed by Neusser et al. were later used to help classify and distinguish between different types of localized and extended modes. ${ }^{9}$ Localized modes are key to understanding a wide range of phenomena in magnonics, including how spin waves propagate in one-dimensional magnetic strips, and details of FMR in individual microstructures. ${ }^{10,11}$ In the present work, we focus on AD edge localized modes residing in a demagnetized region on the edges of the AD. Such a mode was first measured using TRKM by Pechan et al. on an individual AD feature. ${ }^{8}$ It has been suggested that this mode would be difficult to observe in a large ADL because edge roughness and size distribution of $\mathrm{AD}$ would significantly broaden the resonance. ${ }^{9,12}$ More recently, an edge mode has been reported in a system of circular AD with spacing less than $400 \mathrm{~nm}$; however, in that work, the localized regions were closely spaced and the mode extended in space due to the overlap of the resonating regions. ${ }^{13}$ In the present work, we study a square ADL with a lattice constant of $1 \mu \mathrm{m}$; therefore, the localized mode will exhibit less overlap between unit cells, and hence be more difficult to observe due to any broadening from nonuniformities of $\mathrm{AD}$ shape and size. We have also performed micromagnetic simulations of various $\mathrm{AD}$ shapes in order to interpret our data and to make a quantitative statement regarding the strong $\mathrm{AD}$ shape dependence of the mode frequency that has been previously suggested. ${ }^{9}$ It is well known that small changes in the feature shape and edges in magnetic dot and ring lattices strongly effect the mode spectra. ${ }^{14}$ Similarly, we find that for a particular edge localized mode, the frequency is highly dependent upon the applied DC field direction and the $\mathrm{AD}$ shape. We find evidence that an edge "localized" mode can turn into an "extended" edge mode as the in-plane angle between the ADL and the applied DC magnetic field is varied (depending on the shape of the $A D$ ), which may be an important consideration for the transmission of spin wave signals in ADL.

Electron beam lithography (EBL) was used to pattern square lattices of square antidots (width $\mathrm{D}=500 \mathrm{~nm}$, and separation $\mathrm{d}=1000 \mathrm{~nm}$ ) in a permalloy (Py) film of thickness $t=25 \mathrm{~nm}$. ZEP positive resist was spin-coated on a $\mathrm{Si}$ wafer; and after EBL exposure and development, the Py film was deposited using electron beam evaporation, with a base pressure of $10^{-7}$ torr. Final lift-off of the resist was done using N-Methyl-2-pyrrolidone (NMP). The overall dimension of the ADL was $2 \mathrm{~mm} \times 2 \mathrm{~mm}$, and a representative scanning electron microscopy (SEM) image of a sample ADL is shown in Fig. 1.

Our experimental set-up utilized a large area $\left(\sim 1 \mathrm{~mm}^{2}\right)$ broadband meanderline that traverses back and forth directly over an adjacent ADL structure. ${ }^{15}$ The meanderline is connected to an RF generator to broadcast microwaves that are closely coupled to the magnetic sample. The voltage generated across the meanderline is rectified by an RF diode detector. The meanderline sample holder is connected to a probe that is lowered between the pole faces of an electromagnet. A $\mathrm{CW}$ RF signal is transmitted through the meanderline as an in-plane DC magnetic field is swept by a computer controlled bipolar power supply. A pair of Helmholtz coils are wrapped around the pole faces so that a field modulated lock-in detection technique could be employed. To this end, the primary field was modulated with a $20 \mathrm{G}$ AC RMS field at $40 \mathrm{~Hz}$. A computer stores each individual rectified transmission voltage vs. field so that signal averaging can be used to increase the signal-to-noise ratio. Signal averaging was of crucial importance for the measurement of the edge localized modes, as their resonant signals were much weaker than bulk modes. The experiments were repeated for various in-plane field 


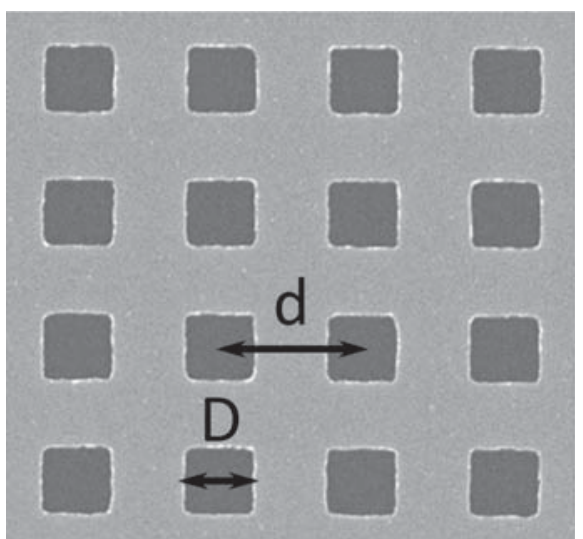

FIG. 1. SEM image of the sample ADL. The AD width is $D=500 \mathrm{~nm}$ and the ADL lattice constant is $d=1000 \mathrm{~nm}$.

angles, $\varphi$, measured relative to the (10) axis of the square ADL.

Broadband data for a DC magnetic field applied along the (10) direction of the array are shown in Fig. 2. At higher frequencies, we observe two large-amplitude resonances first identified by Pechan et al., ${ }^{8}$ along with other modes discussed elsewhere in the literature. ${ }^{9,12}$ These resonances all enter into the region of DC magnetization hysteresis as the RF frequency is lowered. At $2.1 \mathrm{GHz}$ (see Fig. 2), we observed an additional, smaller-amplitude resonance at a relatively high DC field. We consider this to be an edge localized mode candidate, since for a fixed-frequency experiment, one expects the mode to be the highest field resonance. The remaining part of the experimental discussion will be devoted to this mode.

In order to characterize the edge localized mode candidate, we varied the in-plane angle $\varphi$ and recorded the field position of the resonance as a function of $\varphi$. Examples of some of these traces are shown in Fig. 3. As the applied DC field direction was rotated, a second line emerged at $\varphi=10^{\circ}$ at higher field strengths. This is not unexpected as multiple edge localized modes have been observed on a single AD. ${ }^{8}$ The resonance fields of these two modes continue to move downward as $\varphi$ increases. At $\varphi=30^{\circ}$, a third mode emerges at a still higher field. As $\varphi$ increases from $30^{\circ}$ to $40^{\circ}$, the amplitudes of the modes weaken and the lowest-field mode disappears. Finally, at $\varphi=45^{\circ}$, there are only two clear modes remaining. The angular dependence of the mode

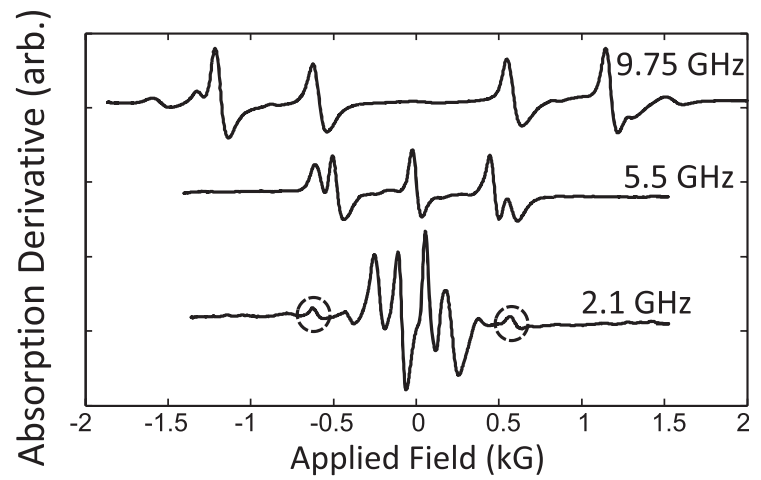

FIG. 2. Resonance spectra as a function of field showing the evolution of ADL modes as a function of frequency. The circled resonances at $2.1 \mathrm{GHz}$ are the edge localized mode candidates.

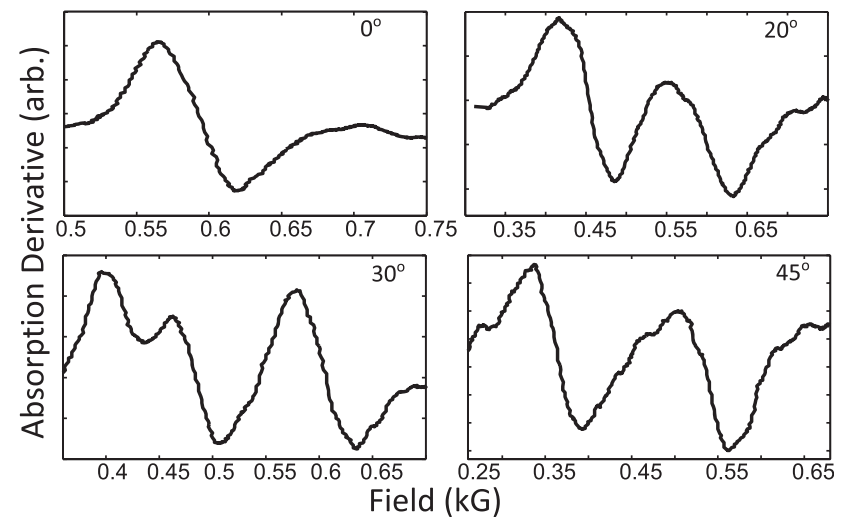

FIG. 3. The angular progression of the candidate signals are shown above. As the field is rotated in-plane not only do the positions change, but more than one resonance can be identified.

frequencies is shown in Fig. 4. We do not distinguish these individual edge mode candidates from one another. The reasons here are two-fold: The resonances seen in Fig. 3 are not well separated, overlapping with one another; the appearance and disappearance of additional resonances are sudden on the angular step size that we use.

Micromagnetic modeling of the modes was performed to corroborate and interpret the experimental data. To this end, a hypothetical, $20 \mathrm{~nm}$ thick, approximately square $8 \mu \mathrm{m} \times 8 \mu \mathrm{m}$ slab with $D=500 \mathrm{~nm}$ and $d=1000 \mathrm{~nm}$ and saturation magnetization, $M_{S}=800 \mathrm{emu} / \mathrm{cm}^{3}$, was divided into a cubic lattice of cells with lattice constant $10 \mathrm{~nm}$. The Landau-Lifshitz-Gilbert (LLG) equation for the time dependent magnetization was integrated for the magnetization director, $\mathbf{m}\left(\mathbf{r}, t_{i}\right)=\mathbf{M}\left(\mathbf{r}, t_{i}\right) / M_{S}$, in each cell in time steps varying from $0.25 \mathrm{ps}$ to $0.5 \mathrm{ps}$.

The magnon frequencies were obtained by first equilibrating the system in an applied external field, and then perturbing it using a short pulse directed approximately perpendicular to the applied static magnetic field. The ensuing time dependence of the magnetization response was then obtained from the LLG equation by integration over a 10-ns interval using a dimensionless damping constant of $\alpha=0.015$. During the time integration, $\mathbf{m}\left(\mathbf{r}, t_{i}\right)$ was averaged spatially over the entire sample at each discrete time step $t_{i}, i=0,1, \ldots N-1$ to obtain time sequences of the average magnetization $\left\langle m_{\alpha}\left(t_{i}\right)\right\rangle, \alpha=x, y, z$, where $\langle\cdots\rangle$ denotes spatial average. These time sequences were then Fourier transformed with respect to time to get the frequencydependent average magnetization, $m_{\alpha}\left(\omega_{i}\right)$. This procedure

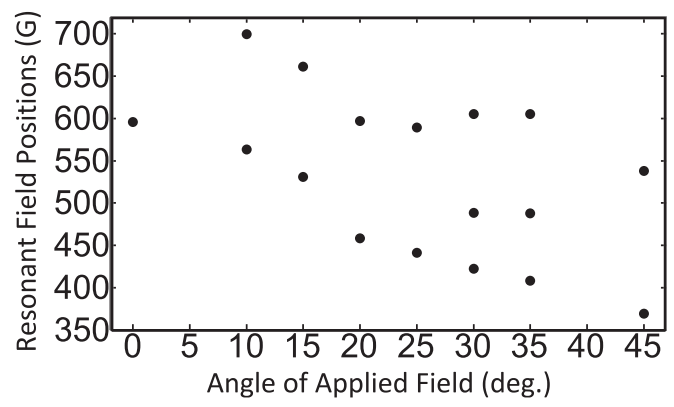

FIG. 4. Experimental in-plane angular dependence of the edge localized mode candidate signals. 
generally gives an accurate representation of the magnon frequencies excited by the initial pulse, with peaks in the Fourier transform corresponding to peaks in the dynamical susceptibility $\chi(\boldsymbol{q} \rightarrow 0, \omega)$.

In order to image the magnetization distribution of the modes, a time sequence of 2D images of the magnetization distributions in the lattice plane was Fourier transformed with respect to time. This process gives $2 \mathrm{D}$ spatial amplitude and phase maps of the dynamical magnetization texture at each (discrete) frequency step $\omega_{i}$, with the frequency resolution determined by the length of the time integration. Open boundary conditions were used at the edges of the $8 \mu \mathrm{m} \times 8 \mu \mathrm{m}$ sample area. This does introduce magnetic charges at the bounding edges of the system that can contribute their own resonance, especially when the external magnetic field is close to $90^{\circ}$ to the edge. In order to minimize interference between these boundary modes from the $8 \mu \mathrm{m} \times 8 \mu \mathrm{m}$ sample and the edge localized modes that are the subject of this study, we performed simulations with the (10) direction of the ADL rotated $45^{\circ}$ with respect to the boundaries of the simulation system. The simulations were performed at fixed field for technical reasons, while the experiments were performed at fixed frequency. Furthermore, as we will show below, the precise spectra and dispersion are very sensitive to the geometrical details of the AD. Therefore, we would not expect to get accurate quantitative agreement between our data and simulations. We note, if one scales the simulated frequencies and fields down using a Kittel-like expression (uniform FMR mode formula), the quantitative agreement between experiment and simulations is quite good.

In order to assess the effects of AD edge topology on the FMR spectra, we briefly discuss the simulated behavior of a square lattice of circular $\mathrm{AD}$ for comparison to the experimental results for square AD presented below. For circular $\mathrm{AD}$, we found an edge localized mode resonance almost independent of the in-plane angle, as shown in Fig. 5, which is consistent with a rotationally invariant $\mathrm{AD}$. As the DC field direction is rotated with respect to the ADL axes from the (10) direction to the (11) direction, the distance between neighboring localized modes increases, which slightly shifts the mode frequencies up from $4.4 \mathrm{GHz}$ to $4.8 \mathrm{GHz}$ at $1 \mathrm{kOe}$ applied field. This is attributed to the demagnetizing fields diminishing as the field is rotated towards the (11) direction. Near $\varphi=10^{\circ}$, the simulations show evidence of two localized edge modes, one at about $4.8 \mathrm{GHz}$, and the other at $5.6 \mathrm{GHz}$. The difference between the modes is a spatial phase modulation (that will be elucidated later), and a slight amplitude modulation of the higher-frequency mode.

Unlike the circular ADL, square ADL will exhibit strong anisotropic shape-dependent demagnetization effects that play a role in the angular dispersion, as shown in Fig. 6. In this case, the angle between the applied field and AD edge of the hole does not remain constant as the field is rotated, which changes the build-up of magnetic charge along the $\mathrm{AD}$ edges. Therefore, if the applied field intersects the AD edges at sharp corners, where the curvature of the AD rapidly changes, one would then expect a rapidly varying demagnetization field, and a concomitant rapid change in mode frequency with $\varphi$. Indeed, micromagnetic modeling at a field strength of $1 \mathrm{kOe}$ shows an edge localized mode at

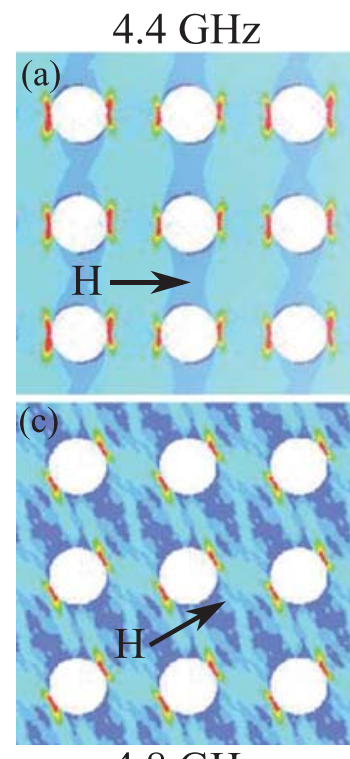

$4.8 \mathrm{GHz}$
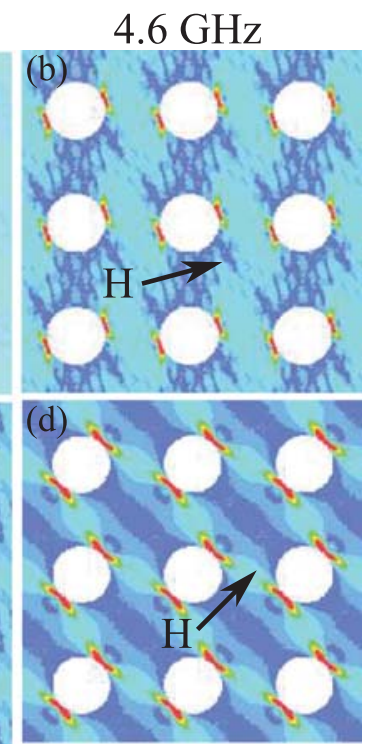

$4.8 \mathrm{GHz}$
FIG. 5. Simulated edge localized modes for a circular ADL for different inplane field directions (a) $0^{\circ}$, (b) $15^{\circ}$, (c) $30^{\circ}$, and (d) $45^{\circ}$. The color scale indicates the precessional amplitude from blue (zero amplitude) to red (high amplitude) of the magnetization. The mode is clearly concentrated near the $\mathrm{AD}$ edges over the entire angular range.

$1.4 \mathrm{GHz}$ at $\varphi=0^{\circ}$ with the large amplitude precession along the edges of the AD squares perpendicular to the field. As $\varphi$ increases, the location of the dynamical magnetization moves rapidly towards the corners of the AD (Fig. 6) and the frequency increases rapidly. Simultaneously, as the mode frequency increases the character of the mode changes from local to extended through the ADL. For perfect squares, the mode frequency increases from $1.4 \mathrm{GHz}$ at $0^{\circ}$ to $8.4 \mathrm{GHz}$ at $45^{\circ}$. In addition, up to four distinct modes appear in a narrow range of angles between $\varphi=0^{\circ}$ and $\varphi=10^{\circ}$.

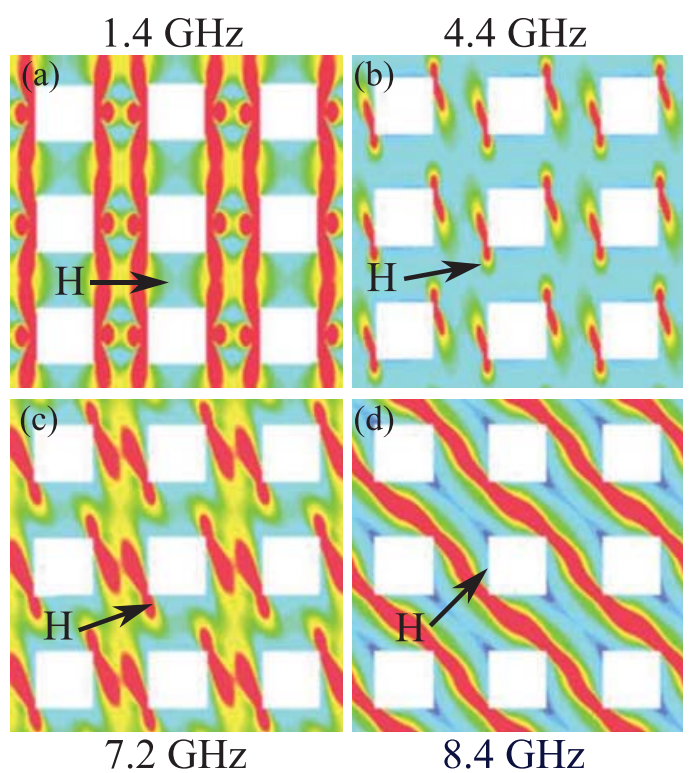

FIG. 6. Simulated edge localized modes for a square ADL for different in-plane field angles of (a) $0^{\circ}$, (b) $10^{\circ}$, (c) $20^{\circ}$, and (d) $45^{\circ}$. Color indicates the precessional amplitude of the resonating regions, with red corresponding to the largest amplitude. As the field is rotated, the character of the mode changes from localized to extended. 


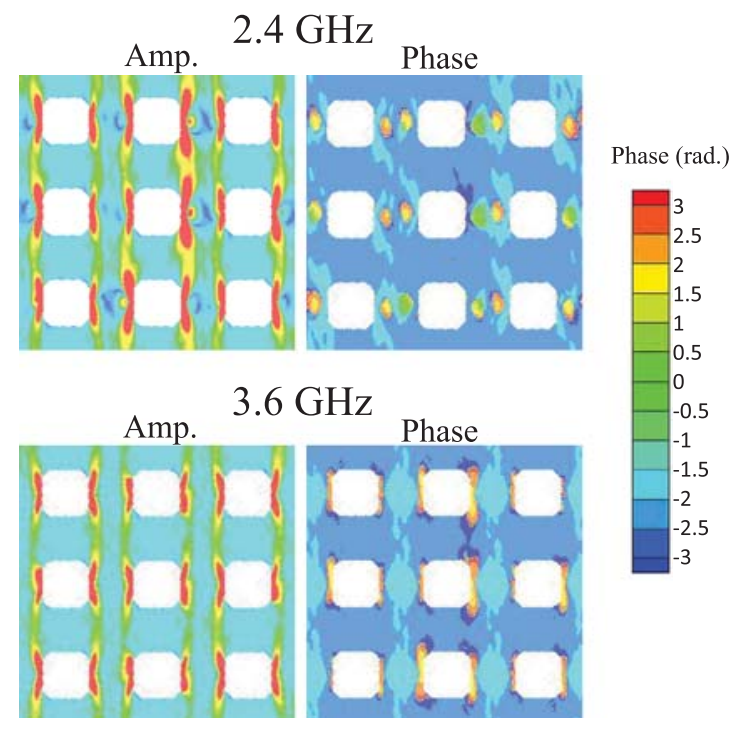

FIG. 7. Simulated edge localized mode magnetization and phase distributions at the $2.4 \mathrm{GHz}$ resonance (top panels) and $3.6 \mathrm{GHz}$ resonance (bottom panels) in $1 \mathrm{kOe}$ field and at $\varphi=0^{\circ}$. Note the different locations of the phase variations in the $2.4 \mathrm{GHz}$ and $3.6 \mathrm{GHz}$ modes (right panels). The phase maps show some numerical noise, but the different signatures of the phases in the two modes is well above the noise level.

The ADs in our experimental arrays have rounded corners that arise from the finite resolution involved in EBL patterning. Compared to the round $\mathrm{AD}$ and the square $\mathrm{AD}$, one would expect square AD with rounded corners to exhibit localized modes that are less sensitive to the in-plane field angle than square $\mathrm{AD}$, and a smaller number of modes than for the square $\mathrm{AD}$. Micromagnetic modeling using a $1 \mathrm{kOe}$ applied field revealed two to three edge localized modes in the range of $0^{\circ} \leq \varphi \leq 30^{\circ}$. At $\varphi=0^{\circ}$, there are two distinct modes at $2.4 \mathrm{GHz}$ and $3.6 \mathrm{GHz}$. We note that this compares rather well with the experimental frequency at $2.1 \mathrm{GHz}$ observed at 596 Oe. While spatial variations in the precessional amplitudes are roughly similar for the two modes, there is clear difference in the behavior of the spatial phase variations between the two modes, as shown in Fig. 7. The lower-frequency mode has a phase variation that is largest in the regions between near-neighbor $\mathrm{AD}$. The higher energy mode has a slight phase variation in the heavily excited edge region; the lower energy mode is more uniform in phase. In the former case, the phase variation is perpendicular to the

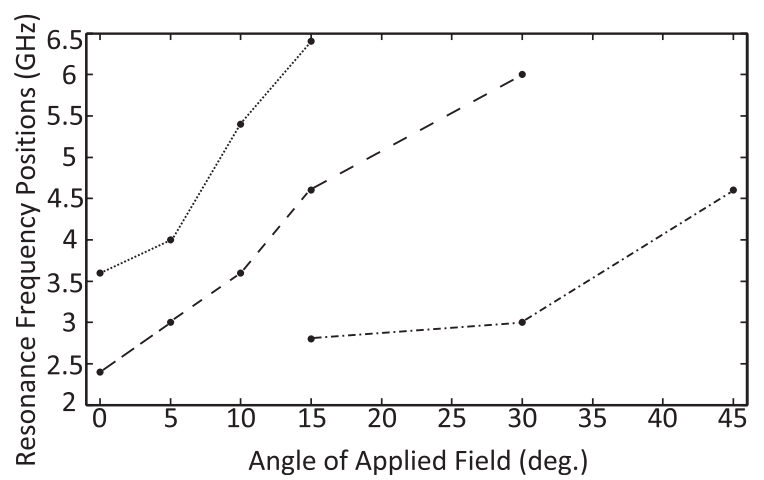

FIG. 8. Micromagnetic edge localized mode frequencies obtained at $1 \mathrm{kOe}$ field for the rounded squares. The dotted lines are plotted as guides for the eyes. applied field; from a magnetostatic viewpoint this will increase the overall energy (along with exchange energy). At $\varphi=5^{\circ}$, there are two discernible resonances at about $2.8 \mathrm{GHz}$ and $4 \mathrm{GHz}$. As $\varphi$ increases, the resonances blueshift in frequency and the lower-frequency modes become weaker. At $\varphi=45^{\circ}$, there is a single clear mode at $4.6 \mathrm{GHz}$ which is very close to the frequency for a round AD. Figure 8 shows all the discernible edge localized mode frequencies obtained from the micromagnetic modeling.

In summary, we have presented experimental and modeling evidence for highly localized edge modes in large ADL. This has been reported to be experimentally difficult to observe. ${ }^{9,12}$ The frequency of the edge localized mode and its topological character are both highly dependent on the shape of the AD. Rounded-square and circular AD are less sensitive to in-plane field angle, with the dynamical magnetization localized at the edge of the hole. The mode in the square AD is very sensitive to the in-plane field angle, both in frequency and in the character of the dynamical magnetization, with the mode becoming extended near $\varphi=0^{\circ}$ and $\varphi=45^{\circ}$. This warrants further investigation as extended modes in magnonic crystals are expected to be useful in the transmission of information via spin waves. By engineering the shape of the $\mathrm{AD}$ hole, varying the field angle may open new avenues of "opening" and "closing" magnonic waveguides by changing the modes from extended to localized in space.

This work received support by the Air Force Office of Scientific Research and NSF supported Northwestern International Institute for Nanoscience. This work utilized facilities maintained by the NSF supported Northwestern Materials Research Center. Research at the University of Kentucky is supported by the U.S. DoE Office of Basic Energy Sciences Grant DE-FGO2-97ER45653. Argonne National Laboratory is operated under Contract No. DEAC02-06CH11357 by UChicago Argonne, LLC.

${ }^{1}$ V. V. Kruglyak, S. O. Demokritov, and D. Grundler, J. Phys. D: Appl. Phys. 43, 264001 (2010).

${ }^{2}$ M. P. Kostylev, A. A. Serga, T. Schneider, B. Leven, and B. Hillebrands, Appl. Phys. Lett. 87, 153501 (2005).

${ }^{3}$ A. Khitun and K. L. Wang, Superlattices Microstruct. 38, 184 (2005).

${ }^{4}$ A. V. Chumak, A. A. Serga, B. Hillebrands, and M. P. Kostylev, Appl. Phys. Lett. 93, 022508 (2008).

${ }^{5}$ A. V. Chumak, A. A. Serga, S. Wolff, B. Hillebrands, and M. P. Kostylev, Appl. Phys. Lett. 94, 172511 (2009).

${ }^{6}$ B. Lenk, H. Ulrichs, F. Garbs, and M. Münzenberg, Phys. Rep. 507, 107-136 (2011).

${ }^{7}$ C. Yu, M. Pechan, and G. Mankey, App. Phys. Lett. 83, 3948-3950 (2003).

${ }^{8}$ M. J. Pechan, C. T. Yu, R. L. Compton, J. P. Park, and P. A. Crowell, J. Appl. Phys. 97, 10J903 (2005).

${ }^{9}$ S. Neusser, B. Botters, and D. Grundler, Phys. Rev. B 78, 054406 (2008).

${ }^{10}$ V. Demidov, S. Demokritov, K. Rott, P. Krzysteczko, and G. Reiss, Appl. Phys. Lett. 92, 232503 (2008).

${ }^{11}$ F. Giesen, J. Podbielski, T. Korn, and D. Grundler, J. Appl. Phys. 97, 10A712 (2005).

${ }^{12}$ S. Neusser, B. Botters, M. Becherer, D. Schmitt-Landsiedel, and D. Grundler, App. Phys. Lett. 93, 122501 (2008).

${ }^{13}$ S. Neusser, G. Duerr, S. Tacchi, M. Madami, M. L. Sokolovskyy, G. Gubbiotti, M. Krawcyzk, and D. Grundler, Phys. Rev. B. 84, 094454 (2011).

${ }^{14}$ W. Xu, D. B. Watkins, L. E. DeLong, K. Rivkin, J. B. Ketterson, and V. V. Metlushko, J. Appl. Phys. 95, 6645 (2004).

${ }^{15}$ C. C. Tsai, J. Choi, S. Cho, S. J. Lee, B. K. Sarma, C. Thompson, O. Chernyashevskyy, I. Nevirkovets, and J. B. Ketterson, Rev. Sci. Instrum. 80, 023904 (2009). 